Check for updates

Cite this: RSC Adv., 2018, 8, 29830

\title{
Investigation on cure kinetics of epoxy resin containing carbon nanotubes modified with hyper- branched polyester
}

\author{
Li Lu, abc Liao Xia, (D) *a Hao Zengheng, ${ }^{\text {bc }}$ Sheng Xingyue, ${ }^{b}$ Zhang Yi ${ }^{\mathrm{b}}$ and Liu Pan ${ }^{\mathrm{b}}$
}

The cure kinetics of epoxy resin cured by diethyltoluene diamine (D-EP), D-EP/multi-walled carbon nanotube (D-EP/CNT) composites and D-EP/hyper branched polyester functionalized CNTs (D-EP/CNTs-H2O) were investigated by non-isothermal differential scanning calorimetry (DSC). Results revealed that the presence of CNTs shifted the cure temperature to a lower temperature and accelerated the curing of D-EP, and the addition of CNTs-H2O exhibited a stronger effect in accelerating the cure of D-EP. Activation energies were calculated based on the Kissinger approach and Ozawa approach respectively. Lowered activation energy was observed after the addition of CNTs or CNTs-H2O at low degrees of cure, indicating that the CNTs had a large effect on the curing reaction. The presence of CNTs facilitated the curing reaction, especially the initial epoxyamine reaction. Moreover, CNTs-H2O exhibited better performance. The autocatalytic model was used to describe the cure kinetics phenomena of the studied systems. When CNTs or CNTs-H2O were added, the Sesták-Berggren model still can describe the cure kinetics of the DEP composites because the results, calculated by the model, agreed with the experimental data well. Moreover, the kinetics parameters as well as the equation describing the cure process were proposed.

Received 28th May 2018

Accepted 18th August 2018

DOI: $10.1039 / c 8 \mathrm{ra04525a}$

rsc.li/rsc-advances high Young's modulus as well as good electrical and thermal conductivity. ${ }^{\mathbf{9} 14-17}$ The addition of a small amount of CNTs can strongly improve the electrical, thermal and mechanical properties of the polymer matrix, resulting in the composites with good processability, balanced stiffness and toughness as well as other functional properties. ${ }^{\mathbf{1 8 - 2 1}}$ However, the main problem is that, the CNTs are insoluble and difficult to be evenly dispersed in the matrix due to its inorganic nature and lack of functional groups on the surface. To improve its compatibility with epoxy resin, the surface modification is proved to be an effective way. ${ }^{11,22-24}$

Hyperbranched polymers have a lower branching efficiency than dendrimers while possessing many useful properties. ${ }^{25-27}$ The highly branched architecture minimizes chain-chain ability and low melt viscosity to these polymers, which is compatible with epoxy thermosets. The presence of plenty of functional groups $(-\mathrm{OH})$ provides high reaction activity with inorganic filler, providing us a possibility to be desirable macromolecular surface modifier for CNTs, to further enhance the compatibility and dispersion in epoxy resin. In the previous study, ${ }^{8}$ we successfully prepared hyperbranched polyester grafted CNTs, and applied it in the toughening of epoxy resin cured by diethyltoluene diamine (DEP). Results revealed that the hyperbranched polyester grafted CNTs received a better dispersion in the epoxy prepolymer compared with the raw CNTs, which further decreased the glass transition temperature of the D-EP/CNTs composites and exhibited better performance in the enhancement in toughening D-EP.

The previous study provides novel method to prepare high performance D-EP/CNTs composites. However, the surface 
modification of CNTs using hyperbranched polyester might influence the curing kinetics, which is very important in understanding the impact of modified CNTs on the performance of D-EP/CNTs composites. The aim of this study is to comparatively investigate the role of hyperbranched polyester surface modification of CNTs in the cure kinetics of the D-EP/ CNTs resin. From the curing kinetics analysis, the better understanding of the impact of CNTs and CNTs-H20 in the curing behavior, morphology and performances of the composites can be obtained.

\section{Experimental}

\subsection{Materials}

The epoxy resin used (Epon® $\left.{ }^{\circledR} 825\right)$ was purchased from Shell (Houston, TX, USA); the carboxylic multi-walled carbon nanotube (denoted as CNTs in this paper) was purchased from Chengdu Organic Chemicals Co., LTD., Chinese Academy of Science, China. The diameter of the raw CNTs ranged from 10 to $20 \mathrm{~nm}$ and their length was about 5-15 $\mu \mathrm{m}$.

Hyperbranched polymer Boltorn $\mathrm{H} 20\left(M_{\mathrm{w}}=1350 \mathrm{~g} \mathrm{~mol}^{-1}\right.$, hydroxyl number $=320 \mathrm{mg} \mathrm{KOH}$ per $\mathrm{g}$ polymer, names as Hyper $\mathrm{H} 20$ in this study) was purchased from Shanghai Seebio Biotech, Inc (China), and was used as received; diglycidyl ether of bisphenol A (DGEBA, Araldite GY 240, Huntsman) (182 g $\mathrm{mol}^{-1}$ ) was dried under vacuum at $80{ }^{\circ} \mathrm{C}$ for $8 \mathrm{~h}$ before use; diethyltoluene diamine (DETDA) and $N, N$-dimethylformamide (DMF) were purchased from Sinopharm Chemical Reagent Beijing Co., Ltd, China, and were used as received.

The chemical structures of the epoxy, DGEBA, DETDA, CNTs and Hyper $\mathrm{H} 20$ are shown in Scheme $1 .^{8}$

\subsection{Preparation of CNTs-H20}

The hyperbranched polyester Hyper H20 and CNTs were dissolved in DMF in room temperature, and then irradiated using ultrasound for $1 \mathrm{~h}$. After that, they were heated up to $120^{\circ} \mathrm{C}$ and react under magnetic stirring for $24 \mathrm{~h}$. After that, they were cooled to room temperature slowly and filtered using PVDF filtration membrane with the average pore diameter of $0.22 \mu \mathrm{m}$. The product was firstly washed by acetone and filtered for several times to eliminate the unreacted Hyper H20 and extra solvent. Each time, the washed acetone was taken for FT-IR analysis, and the washing is finished until the washed acetone has the same FT-IR spectra compared with pure acetone. The collected material was then heated under vacuum drier at $80{ }^{\circ} \mathrm{C}$ for $24 \mathrm{~h}$, and the final product, CNTs grafted with Hyper H20 (named as CNTs-H20), was obtained. The reaction principle between Hyper H20 and CNTs was shown in Scheme 2. ${ }^{8}$

\subsection{Preparation of D-EP/CNTs and D-EP/CNTs-H20 composites}

To prepare D-EP/CNTs composites for the DSC measurement, the epoxy prepolymer and CNTs (CNTs or CNTs-H20) were firstly mixed in the weight ratio of $99: 1$, then, they were irradiated for $1 \mathrm{~h}$ to ensure a finely dispersion of CNTs in epoxy prepolymer. After that, $20 \mathrm{wt} \%$ DETDA were added, and the mixture was used for thermal analysis. Three samples used in this study were named as D-EP, D-EP/CNTs and D-EP/CNTs-H20, respectively.

\subsection{Characterization}

2.4.1 FT-IR. A Nicolet iS50 FTIR spectrometer was used to determine the FTIR spectra of the samples. The FTIR was used at a resolution of $4 \mathrm{~cm}^{-1}$ and 20 scans were averaged for each spectrum. The scanning range was $4000-400 \mathrm{~cm}^{-1} \cdot{ }^{28}$

2.4.2 Transmission electron microscopy (TEM). To study the morphologies of the CNTs and CNTs-H20, transmission electron microscopy (TEM, Tecnai G2 F20, FEI, USA) was applied. $^{29}$

2.4.3 Differential scanning calorimetry (DSC). Dynamic DSC analysis was performed by METTLER TOLEDO DSC1 (METTER TOLEDO, Swiss) which was calibrated with high purity indium and zinc standards. ${ }^{30,31}$ The samples (4-5 mg) were added to aluminum pans and analyzed dynamically with heating rates at $5,10,15$, and $20 \mathrm{~K} \mathrm{~min}^{-1}$ in nitrogen atmosphere. Nitrogen was purged at rate of $40 \mathrm{~mL} \mathrm{~min}^{-1}$ to minimize oxidation of the sample. Measurements were carried out with an empty cell as reference from 300 up to $600 \mathrm{~K}$.

\section{Results and discussion}

\subsection{Characterization of the CNTs and CNTs-H20}

The microstructure of CNTs and CNTs-H20 have been characterized in detail in the previous study. ${ }^{8} \mathrm{~A}$ brief description is given here. The FT-IR spectra of CNTs, CNTs-H20 and Hyper $\mathrm{H} 20$ are shown in Fig. 1.

Fig. 1 shows that for Hyper $\mathrm{H} 20$, the peak at $3420 \mathrm{~cm}^{-1}$ represents the vibration of $-\mathrm{OH}$ group, while signals at the wavenumbers of $2921 \mathrm{~cm}^{-1}$ and $2888 \mathrm{~cm}^{-1}$ represent the asymmetric and symmetric vibrations of $-\mathrm{CH}_{3}$ and $-\mathrm{CH}_{2}$, respectively. The peaks at the wavenumber of $1734 \mathrm{~cm}^{-1}$ is the stretching vibration peak of carbonyl; for CNTs, the $\mathrm{C}=\mathrm{O}$ of carbonyl is in the form of carboxylic acid, the corresponding absorption peak $\left(1734 \mathrm{~cm}^{-1}\right)$ is usually big due to the formation of hydrogen bond; for CNTs-H20, the new peaks at $2921 \mathrm{~cm}^{-1}$ and $2888 \mathrm{~cm}^{-1}$ can be seen, corresponding to the asymmetric and symmetric vibrations of $-\mathrm{CH}_{3}$ and $-\mathrm{CH}_{2}$, respectively, suggesting that the Hyper $\mathrm{H} 20$ is successfully grafted onto CNTs. Moreover, it can also be observed that in the FT-IR spectra of CNTs-H20, the intensity of the peak at $1729 \mathrm{~cm}^{-1}$ representing the stretching vibration peak of carbonyl decreases significantly, indicating that the $-\mathrm{COOH}$ groups on the surface of CNTs have reacted with the terminal hydroxyl groups on hyperbranched polyester (Hyper H20), resulting in the significant decrease of carbonyl absorption peak at $1734 \mathrm{~cm}^{-1}$. Since the un-grafted $\mathrm{H} 20$ has already been washed away before the FTIR test, therefore, the results above suggest that the reaction between $\mathrm{H} 20$ and CNTs takes place, and the reaction mechanism between CNTs and Hyper H20 should be the occurrence of esterification reaction between the carboxyl groups on the surface of CNTs and the hydroxyl groups of Hyper H20.

The TEM images of CNTs and CNTs-H20 are shown in Fig. 2. It can be seen from Fig. 2 that, the morphologies of CNTs and 
CNTs-H20 are quite different from each other. For CNTs, the shape of typical "nanotube" 10 can be clearly seen, while for CNTs-H20, uniform dark-colored morphology can be observed, indicating that the CNTs is covered with Hyper-H20.

\subsection{Basic assumptions of curing kinetics analyzed by DSC}

Using DSC, the curing kinetics of the samples can be characterized by measuring heat signal generated during the curing reaction as a function of temperature and time. ${ }^{22}$ The area under the curves is proportional to the conversion, which could be defined as eqn (1). The heat evolution recorded by DSC is assumed to be proportional to the extent of consumption of the reactive groups. The curing rate during the curing process can be described as the following eqn (2):

$$
\alpha=\frac{H_{t}}{H_{\mathrm{u}}}
$$<smiles>CC(C)(c1ccc(OCC2CO2)cc1)c1ccc(OCC2CO2)cc1</smiles>

Diglycidyl ether of bisphenol A (DGEBA)<smiles>CCc1cc(C)c(N)c(CC)c1N</smiles><smiles>CCc1cc(CC)c(N)c(C)c1N</smiles>

Diethyltoluene diamine(DETDA)

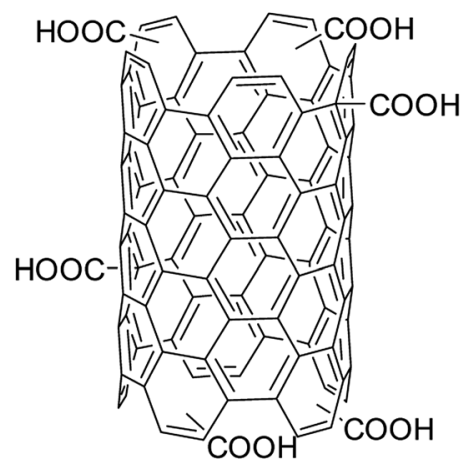

Carboxylic carbon nanotube (CNTs)

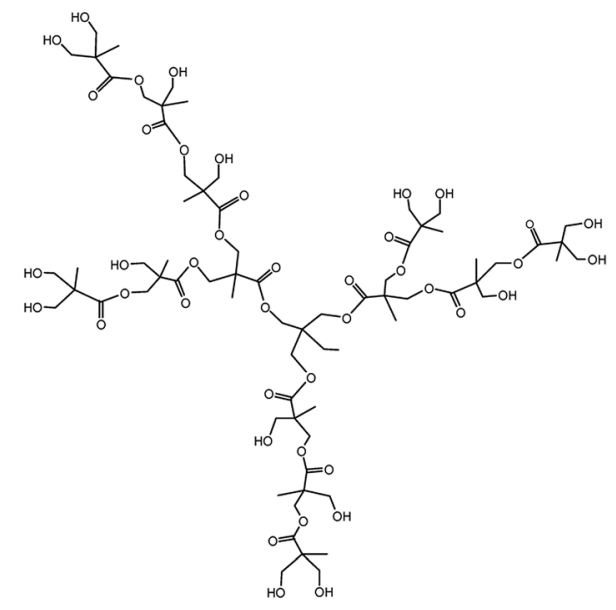

Hyperbranched Polyester (Hyper H20)

Scheme 1 Structure of epoxy resin, curing agent, carbon nanotube and hyperbranched polyester. ${ }^{8}$ 


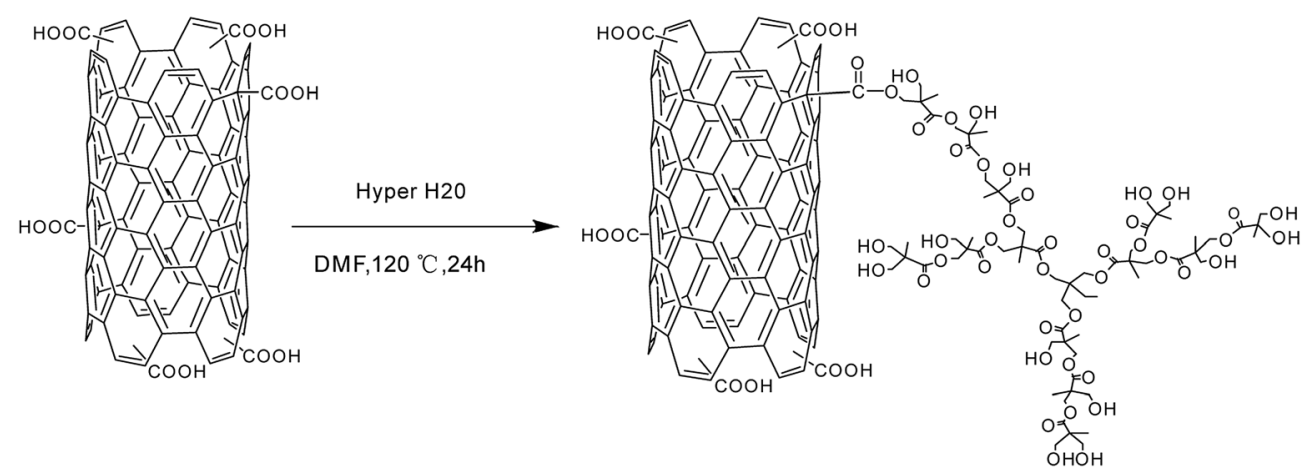

Scheme 2 Reaction principle of hyperbranched polyester modified carbon nanotubes. ${ }^{8}$

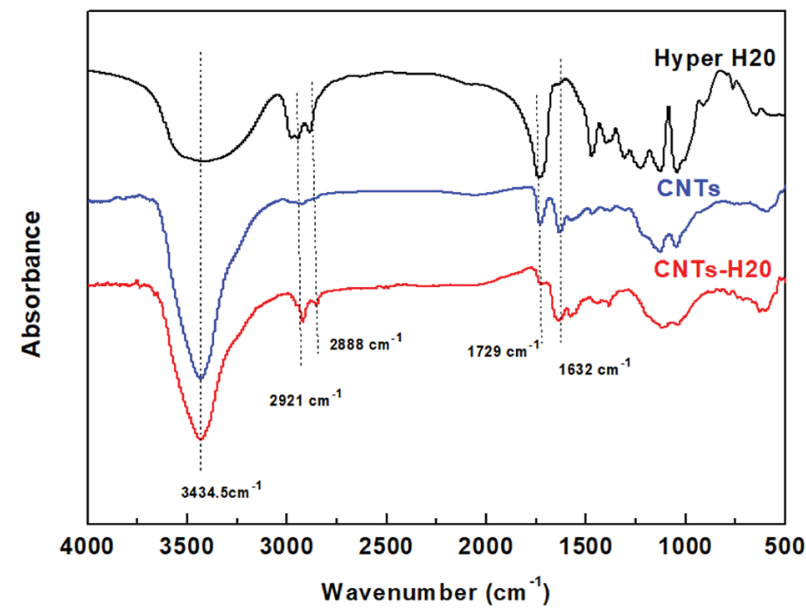

Fig. 1 FT-IR spectra of Hyper H2O, CNTs and CNTs-H2O. ${ }^{8}$

$$
\frac{\mathrm{d} \alpha}{\mathrm{d} t}=\left(\frac{1}{H_{\mathrm{u}}}\right) \frac{\mathrm{d} H}{\mathrm{~d} t}
$$

where $\alpha$ is the extent of curing reaction, $\mathrm{d} \alpha / \mathrm{d} t$ is the curing rate, $H_{t}$ is the reaction heat within time $t, \mathrm{~d} H / \mathrm{d} t$ is the heat flow rate, and $H_{\mathrm{u}}$ is the total heat of reaction.

Most curing kinetics can be described by eqn (3):

$$
\frac{\mathrm{d} \alpha}{\mathrm{d} t}=A \exp \left(\frac{-E_{\mathrm{a}}}{R T}\right) f(\alpha)
$$

where $\mathrm{d} \alpha / \mathrm{d} t$ is the rate of conversion, $A$ is the pre-exponential factor, $E_{\mathrm{a}}$ is the activation energy, $R$ is the gas constant, $T$ is the absolute temperature and the function representing the kinetic model. ${ }^{32}$

The non-isothermal kinetic model can identify two types of reaction: $n$-th order or autocatalytic model. Autocatalytic model (Sesták-Berggren equation) assumes that the reaction obeys the eqn (4): $:^{32}$

$$
\frac{\mathrm{d} \alpha}{\mathrm{d} t}=A \exp \left(\frac{-E_{\mathrm{a}}}{R T}\right) \alpha^{m}(1-\alpha)^{n}
$$

where $m$ is the order of autocatalytic reaction, $n$ is the order of reaction with curing agent. The kinetic equation is widely used in literatures for epoxy systems. In non-isothermal conditions, heating rate $\beta=\mathrm{d} T / \mathrm{d} t$, combined with eqn (4), the kinetic model can be described as follows:

$$
\frac{\mathrm{d} \alpha}{\mathrm{d} T}=\frac{\mathrm{d} \alpha}{\beta \mathrm{d} t}=\frac{A}{\beta} \exp \left(-\frac{E_{\mathrm{a}}}{R T}\right) \alpha^{m}(1-\alpha)^{n}
$$

\subsection{Curing characteristics for the D-EP, D-EP/CNTs and D- EP/CNTs-H20}

DSC curves of the D-EP, D-EP/CNTs and D-EP/CNTs-H20 at different heating rates are shown in Fig. 3. The curing parameters are listed in Table 1 . The conversion $\alpha(T)$ can be calculated by eqn (1). Fig. 3 shows $\alpha(T)$ with respect to $T$ for D-EP, D-EP/ CNTs and D-EP/CNTs-H20 in different heating rates. These curves can be used as basic data for the kinetic calculation.

The polymerization of thermosets is a complex mechanism that may include several chemical reactions. Aromatic primary
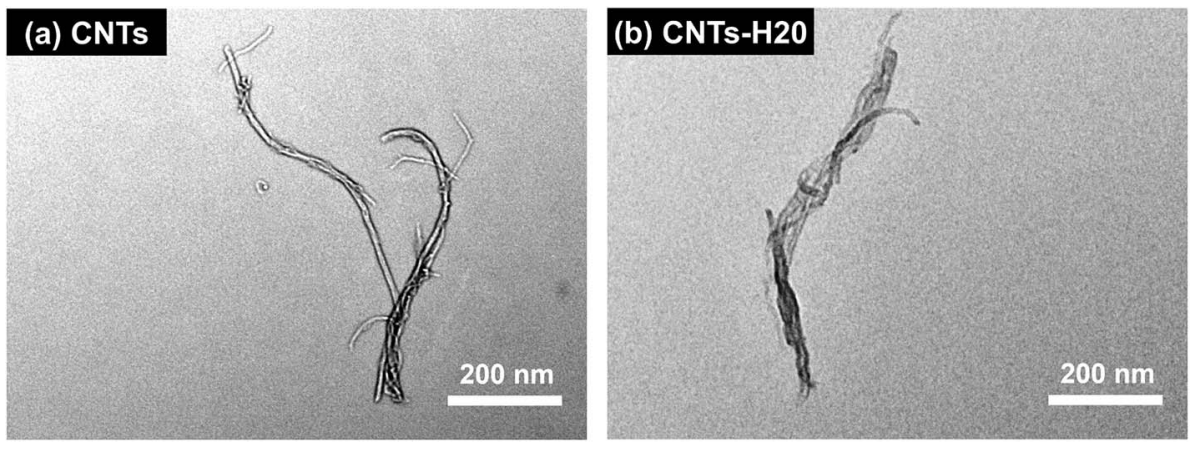

Fig. 2 TEM images of the (a) CNTs and (b) CNTs-H2O. 

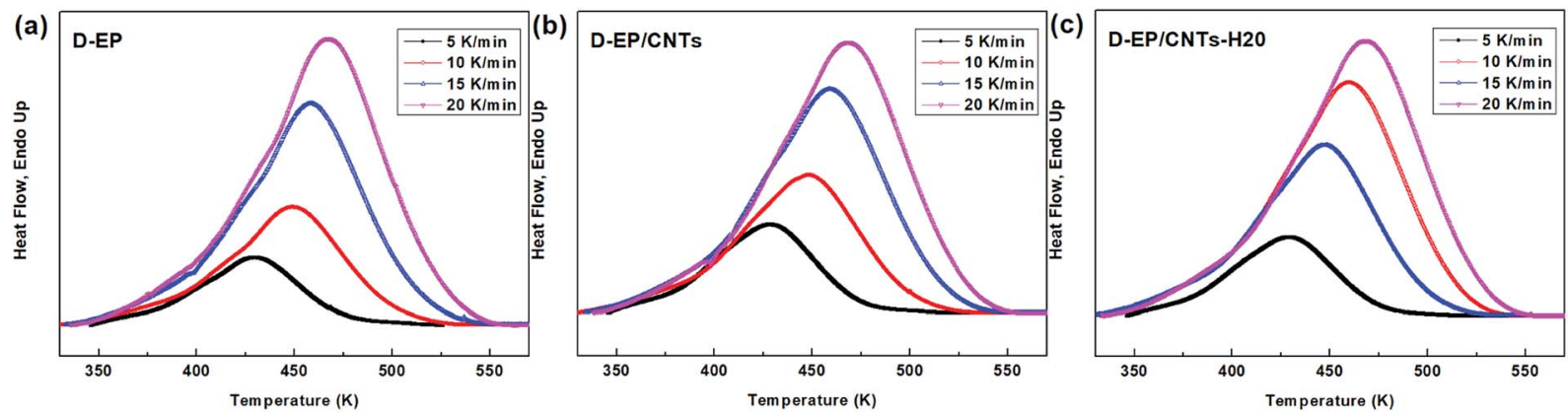

Fig. 3 DSC curing curves of D-EP, D-EP/CNTs and D-EP/CNTs-H2O at different heating rates.

Table 1 Curing characteristics for the D-EP, D-EP/CNTs and D-EP/CNTs-H2O

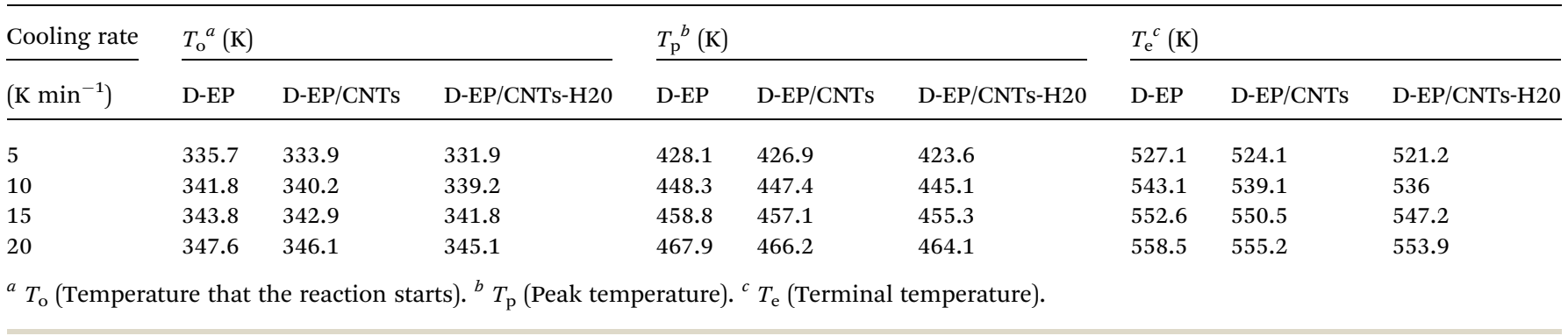
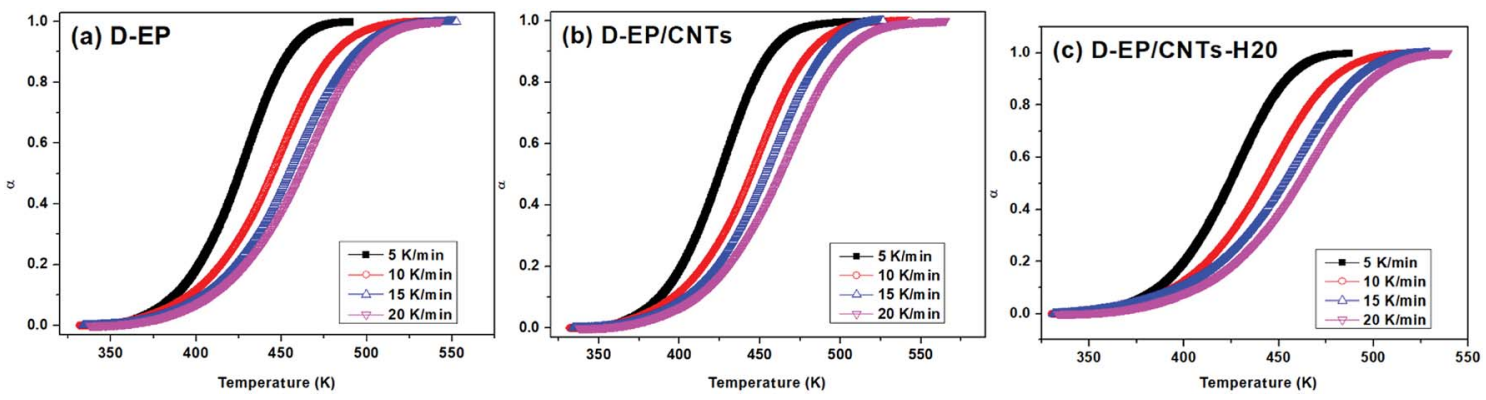

Fig. 4 Conversion $\alpha$ as a function of temperatures for the system of D-EP, D-EP/CNTs and D-EP/CNTs-H2O at different heating rates.

amines are commonly used as curing agents. These active hydrogen compounds undergo an addition reaction with epoxy ring. The polyaddition occurs between epoxy and primary amine, followed by secondary amine addition, and at latter stages of the reaction or at higher temperature, side reactions such as etherification or homopolymerization. It can be seen from the results above that, the presence of CNTs or CNTs-H20 has strong impact on the curing process as reflected by the difference in the DSC curves of the epoxy and its nanocomposites. The presence of CNTs shifts the $T_{\mathrm{o}}, T_{\mathrm{e}}$ and $T_{\mathrm{p}}$ to lower temperature, indicating that the addition of CNTs accelerates the curing of $\mathrm{EP}$, which might be attributed to an increase in the efficiency of collisions, and can be explained by catalytic effect of CNTs on the epoxy ring opening. In other words, the amine groups on the surface of CNTs can play the role of curing agents and promote the primary amine-epoxide reaction..$^{10,11} \mathrm{In}$ addition, a change of the shape of thermo analytical curves suggests possible modifications of the kinetic parameters and of the cure mechanism in the presence of CNTs (Fig. 4).

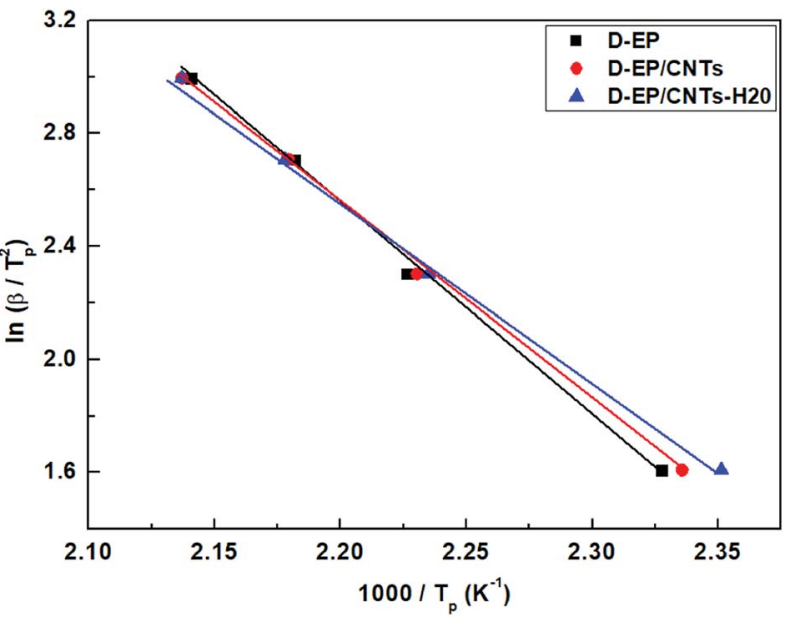

Fig. 5 Plots of $\ln \left(\beta / T_{p}{ }^{2}\right)$ and $1000 / T_{p}$ for the D-EP, D-EP/CNTs and DEP/CNTs-H2O. 

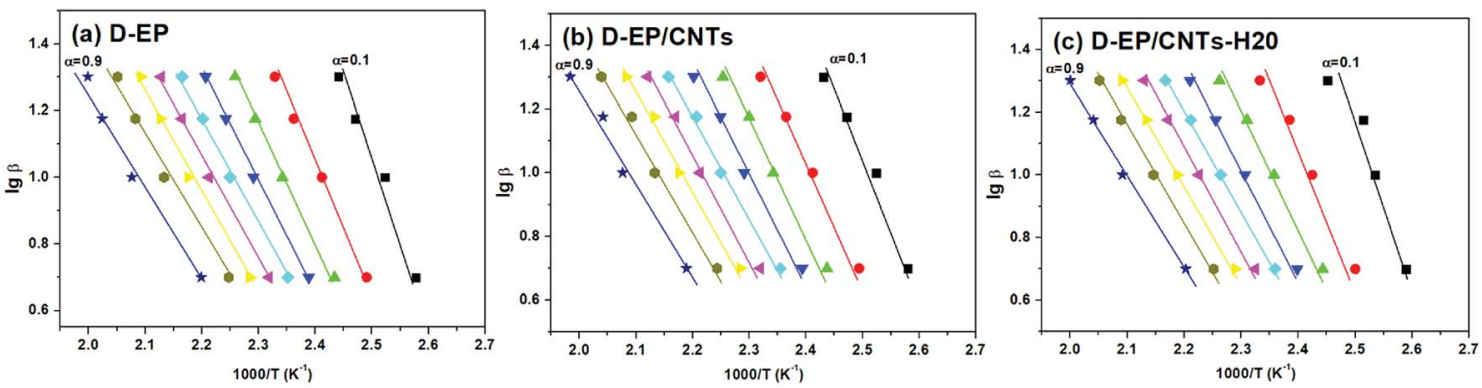

Fig. 6 Isoconversional plots at various conversions for D-EP, D-EP/CNTs and D-EP/CNTs-H2O.

On the other hand, compared with CNTs, it can be observed that after the addition of CNTs-H20, the parameters of $T_{\mathrm{p}}, T_{\mathrm{o}}$ and $T_{\mathrm{e}}$ shift to lower temperature range, indicating that the functionalization of CNTs further accelerates the curing of EP.

\subsection{Calculation of the activation energy of curing}

3.4.1 Kissinger method. Activation energy is usually evaluated by the following two methods: the Kissinger equation and the Ozawa (iso-conversional) equation. ${ }^{2}$ From the Kissinger equation, overall activation energy can be calculated by eqn (6). For non-isothermal curing, the relationship between activation energy $E_{\mathrm{a}}$, the heating rate $\beta$ and the temperature $T_{\mathrm{p}}$ at which the exothermic peak has its maximum can be described as:

$$
\ln \left(\frac{\beta}{T_{\mathrm{p}}^{2}}\right)=\ln \frac{A R}{E}-\frac{E_{\mathrm{a}}}{R T_{\mathrm{p}}}
$$

where $R$ is the gas constant, equal to $8.3144 \mathrm{~J}\left(\mathrm{~mol}^{-1} \mathrm{~K}^{-1}\right)$. From the dynamic DSC curves measured at different heating rates, the relation between $\ln \left(\beta T_{\mathrm{p}}{ }^{-2}\right)$ and $T_{\mathrm{p}}{ }^{-1}$ can be obtained, and then the activation energy can be calculated from the slope.

Fig. 5 shows the relationship between $\ln \left(\beta / T_{\mathrm{p}}{ }^{2}\right)$ and $1000 / T_{\mathrm{p}}$ for both the D-EP system and its nanocomposite. The four points correspond to the four heating rates of $5,10,15$, and 20 $\mathrm{K} \mathrm{min}^{-1}$. Fig. 5 suggests that there is an excellent linear fit in all the cases, indicating that the experimental data fits the Kissinger model quite well. From the slopes of these three plots the overall activation energy for D-EP, D-EP/CNTs and D-EP/CNTs$\mathrm{H} 20$ were found to be $60.6,50.8$ and $47.4 \mathrm{~kJ} \mathrm{~mol}^{-1}$, respectively, indicating that addition of CNTs or CNTs-H20 decreases the activation energy barrier of EP during curing process, making the occurrence of cure easier. The addition of CNTs$\mathrm{H} 20$ further decreases the activation energy compared with CNTs, which might be attributed to the positive effect of the organic intercalant to promote the chemical reaction of CNTsH20. ${ }^{11,26,32,33}$

3.4.2 Ozawa method. Unlike the Kissinger approach, the Ozawa approach makes it possible to determine the activation energy corresponding to different stages of cure throughout the entire conversion. The equation used is: ${ }^{34}$

$$
\lg \beta=\lg \left(\frac{A E}{R G(\alpha)}\right)-2.315-0.4567 \frac{E}{R T}
$$

where $\alpha$ is the extent of curing reaction, $T$ is the temperature corresponding to a selected degree of conversion at a given heating rate $\beta$. From the slope of a plot of $\log \beta v s . T^{-1}$ for a chosen degree of conversion, the activation energy corresponding to that degree of conversion can be obtained.

Fig. 6 shows the relationship between $\log \beta$ vs. $T^{-1}$ for nine different degrees of conversion ranging from 0.1 to 0.95 . The linear relationship observed in all cases indicates that the approach is applicable for this case. The activation energies $E_{\mathrm{a}}$ calculated for the systems are given in Fig. 7 .

As can be seen from Fig. 6 and 7, there is a significant difference in behavior between D-EP, D-EP/CNTs and D-EP/ CNTs-H20: for the D-EP, the apparent $E_{\mathrm{a}}$ keeps more or less steadily as a function of the degree of cure, from $81.0 \pm$ $2.6 \mathrm{~kJ} \mathrm{~mol}^{-1}$ at 0.1 to $41.5 \pm 2.0 \mathrm{~kJ} \mathrm{~mol}^{-1}$ at 0.9 . For the D-EP/ CNTs system, at a low degree of cure $(\alpha<0.3)$ the apparent $E_{\mathrm{a}}$ is obviously lower than the D-EP system, but as the degree of cure increases, $E_{\mathrm{a}}$ decreases slightly, reaching $56.1 \pm$ $1.9 \mathrm{~kJ} \mathrm{~mol}^{-1}$ at $\alpha=0.3$ and then keeps almost constant to $\alpha=$ 0.9. For D-EP/CNTs-H20 system, the $E_{\mathrm{a}}$ is $61.1 \pm 3.2 \mathrm{~kJ} \mathrm{~mol}^{-1}$ at low degree of cure $(\alpha<0.3)$, which is obviously lower than that of D-EP and D-EP/CNTs; which gradually decreases to 52$55 \mathrm{~kJ} \mathrm{~mol}^{-1}$ and keeps almost constant to the finish of cure. The variation of the $E_{\mathrm{a}}$ during the cure process of the samples can be explained as following:

In the early stages of the cure, the temperature is lower, the dominant reaction is expected to be that between epoxy and

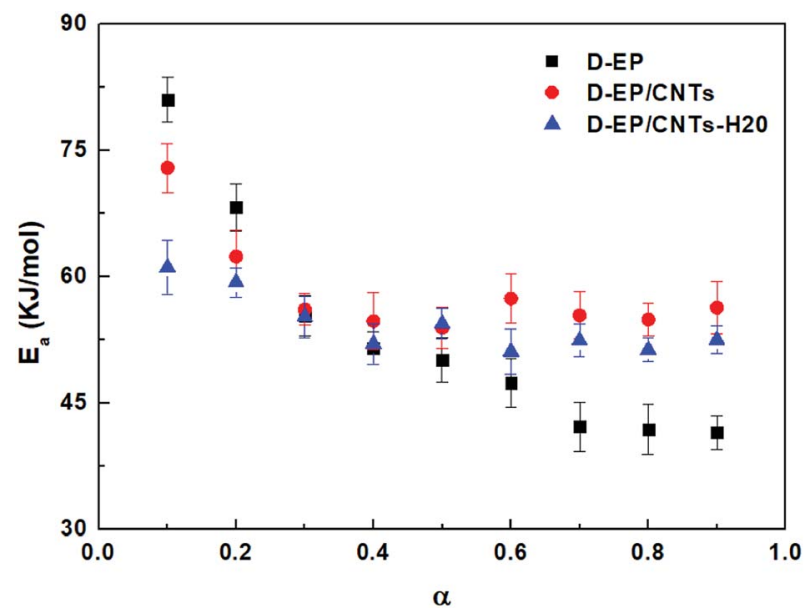

Fig. 7 Variation of $E_{\mathrm{a}}$ versus $\alpha$ by Ozawa method. 

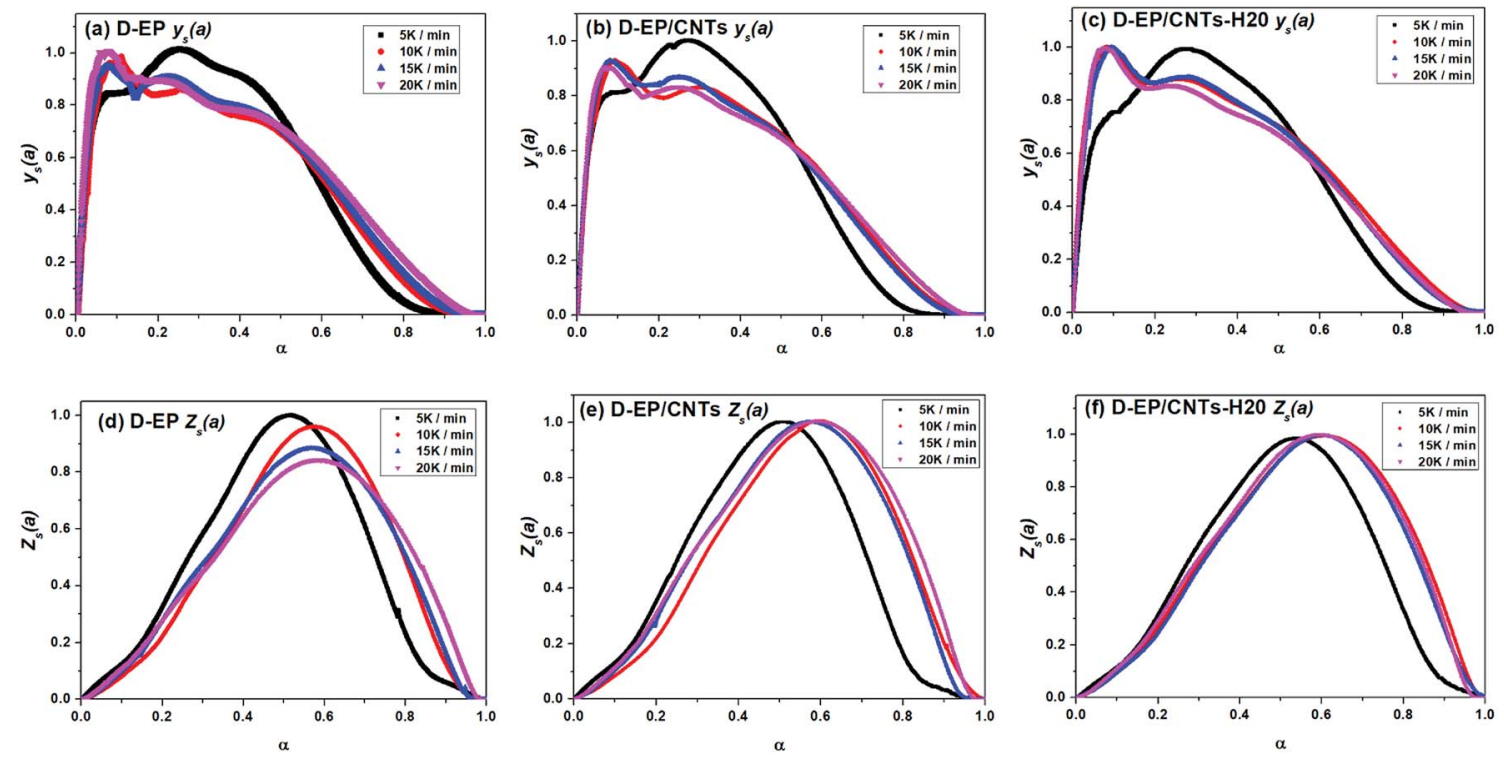

Fig. $8 y_{s}(\alpha)$ and $z_{s}(\alpha)$ functions of D-EP, D-EP/CNTs and D-EP/CNTs-H2O

amine groups. In the later stages of cure, at higher temperatures, other reactions (mainly epoxy etherification, but possibly others like hydroxyl hydroxyl) are expected to become more important. ${ }^{33,35}$ The unusual low activation energy observed for the nanocomposite at lower degrees of cure indicates that the addition of CNTs or CNTs-H20 facilitates the curing reaction, especially the initial epoxy-amine reaction.

The first stage of the dependency of D-EP is characteristic of epoxy-amine reactions. At the end of the reaction, the decrease of $E_{\mathrm{a}}$ is due to the transition from chemical control of the crosslinking to a diffusion control that becomes detectable in this system already at gelation. In the case of D-EP/CNTs and DEP/CNTs-H20 systems, the characteristic decrease of $E_{\mathrm{a}}$ observed is lower, leading to the hypothesis that diffusion contribution is less pronounced in this case. Side reactions (etherifications/homopolymerizations) are known to occur at elevated temperatures, so it can also be postulated that these reactions become significant in the case of D-EP/CNTs-H20.

Moreover, the activation energies obtained by the Kissinger approach (60.6 kJ mol ${ }^{-1}$ for D-EP, $50.8 \mathrm{~kJ} \mathrm{~mol}^{-1}$ and $47.4 \mathrm{~kJ} \mathrm{~mol}^{-1}$ for D-EP/CNTs and D-EP/CNTs-H20 respectively) are lower than those obtained by the Ozawa approach $(63.2,58.2$ and $54.3 \mathrm{~kJ} \mathrm{~mol}^{-1}$ for D-EP, D-EP/CNTs and D-EP/CNTs-H20, respectively). This can be explained by the differences in assumptions and mathematical approach. However, for the two methods, similar results that the apparent slight increase in $E_{\text {a }}$ are observed for the nanocomposites. Thus, both the two approaches can be used to detect the activation energy.

3.4.3 Málek method. In Málek method, once activation energy is determined, two characteristic functions, $y(\alpha)$ and $z(\alpha)$ are defined to find the kinetic model and the kinetic parameters. ${ }^{13}$ In non-isothermal conditions, the two functions can be described as follows:

$$
y(\alpha)=\left(\frac{\mathrm{d} \alpha}{\mathrm{d} T}\right) \beta \exp (x)
$$

$$
z(\alpha)=\pi(x)\left(\frac{\mathrm{d} \alpha}{\mathrm{d} T}\right) T
$$

where $x$ is the reduced activation energy $\left(E_{\mathrm{a}} / R T\right)$ and $\pi(x)$ is the function of temperature integrals whose values can be approximated with sufficient accuracy with a fourth-order rational expression of Liang et al.: ${ }^{13}$

$$
\tau(x)=\frac{x^{3}+18 x^{2}+88 x+96}{x^{4}+20 x^{3}+120 x^{2}+240 x+120}
$$

The values of both $y(\alpha)$ and $z(\alpha)$ functions normalized within $(0,1)$ intervals are given by the following equations:

$$
\begin{aligned}
& y_{\mathrm{s}}(\alpha)=\frac{y(\alpha)}{\max [y(\alpha)]} \\
& z_{\mathrm{s}}(\alpha)=\frac{z(\alpha)}{\max [z(\pi)]}
\end{aligned}
$$

According the shape and the maximum of both $y_{\mathrm{s}}(\alpha)$ and $z_{\mathrm{s}}(\alpha)$ functions, the most suitable kinetic model can be determined among several models and subsequently the kinetic

\begin{tabular}{|c|c|c|c|c|c|c|}
\hline \multirow{2}{*}{$\frac{\beta}{\left(\mathrm{K} \mathrm{min}^{-1}\right)}$} & \multicolumn{2}{|l|}{ D-EP } & \multicolumn{2}{|c|}{ D-EP/CNTs } & \multicolumn{2}{|c|}{ D-EP/CNTs-H20 } \\
\hline & $\alpha_{\mathrm{M}}$ & $\alpha_{\mathrm{p}}^{\infty}$ & $\alpha_{\mathrm{M}}$ & $\alpha_{\mathrm{p}}^{\infty}$ & $\alpha_{\mathrm{M}}$ & $\alpha_{\mathrm{p}}^{\infty}$ \\
\hline 5 & 0.2526 & 0.5155 & 0.2714 & 0.5071 & 0.2772 & 0.5373 \\
\hline 10 & 0.0762 & 0.5729 & 0.0924 & 0.5678 & 0.0792 & 0.5953 \\
\hline 15 & 0.0748 & 0.5658 & 0.0792 & 0.5860 & 0.0951 & 0.6009 \\
\hline 20 & 0.1111 & 0.5846 & 0.0850 & 0.5895 & 0.0835 & 0.5938 \\
\hline Mean & 0.1287 & 0.5597 & 0.1320 & 0.5626 & 0.1338 & 0.5818 \\
\hline
\end{tabular}
parameters such as $n$ and $m$ can be calculated. Fig. 8 shows the variation of $y_{\mathrm{s}}(\alpha)$ and $z_{\mathrm{s}}(\alpha)$ values with conversion $\alpha$.

Table 2 The values of $\alpha_{\mathrm{M}}$ and $\alpha_{\mathrm{p}}^{\infty}$ obtained from the $y_{\mathrm{s}}(\alpha)$ and $z_{\mathrm{s}}(\alpha)$ curves 
Table 3 The kinetic parameters for Sesták-Berggren model by Málek method of the samples

\begin{tabular}{lllll}
\hline Samples & $E_{\mathrm{a}}\left(\mathrm{kJ} \mathrm{mol}^{-1}\right)$ & $m$ & $n$ & $f(\alpha)$ \\
\hline D-EP & $60.6 \pm 0.99$ & $0.44 \pm 0.02$ & $2.98 \pm 0.02$ & $\alpha^{0.44}(1-\alpha)^{2.98}$ \\
D-EP/CNTs & $50.8 \pm 1.13$ & $0.43 \pm 0.02$ & $2.81 \pm 0.02$ & $\alpha^{0.43}(1-\alpha)^{2.81}$ \\
D-EP/CNTs-H20 & $47.4 \pm 0.87$ & $0.35 \pm 0.02$ & $2.25 \pm 0.02$ & $\alpha^{0.35}(1-\alpha)^{2.25}$
\end{tabular}

The curves of $y_{\mathrm{s}}(\alpha)$ and $z_{\mathrm{s}}(\alpha)$ exhibit maxima at $\alpha_{\mathrm{M}}$ and $\alpha_{\mathrm{p}}^{\infty}$, respectively. The values of $\alpha_{\mathrm{M}}$ and $\alpha_{\mathrm{p}}^{\infty}$ are shown in Table 2. The results in Fig. 8 and Table 2 indicate the curing process can be described by the two-parameter autocatalytic kinetic model Sesták-Berggren (eqn (5)). ${ }^{13}$ The mean value of $\alpha_{M}$ was used to calculate the kinetic parameters. In eqn (5), the ratio of reaction order, $p=m / n$ as pointed out by Málek, can be replaced by:

$$
p=\frac{m}{n}=\frac{\alpha_{\mathrm{Mm}}}{1-\alpha_{\mathrm{Mm}}}
$$

where $\alpha_{\mathrm{Mm}}$ is the mean of $\alpha_{\mathrm{M}}$. The kinetic parameters $n$ is obtained from the slope of $\ln \left[(\mathrm{d} \alpha / \mathrm{d} T) \beta \mathrm{e}^{x}\right] v s \cdot \ln \left[\alpha^{\mathrm{P}}(1-\alpha)\right]$ (from eqn (4), (5) and (13)) as well as the kinetic parameter $m$ can be calculated from eqn (13).

All of kinetic parameters, $E, n$ and $m$ are listed in Table 3. The validity of Málek method is evaluated by comparison of the curves of $\mathrm{d} \alpha / \mathrm{d} T$ versus $T$ plotted with the calculated data in Table 3 and experimental data. Compared results for D-EP, DEP/CNTs and D-EP/CNTs-H20 are shown in Fig. 9.
The results in Fig. 9 show that calculated data coincides with experimental data, indicating that the Sesták-Berggren model deduced by Málek method could describe the entire curing reaction process. On the other hand, the results in Table 3 reveal that after the addition of CNTs, the reaction order parameters $m$ and $n$ changes evidently, reflecting the significant influence of CNTs and the modification of CNTs on the curing behavior of the epoxy resin. Moreover, the parameters obtained above can be used to describe the curing kinetics (to calculate the degree of cure at certain time certain temperature, to calculate the time or temperature needed to achieve certain degree of cure) according to following equation:

$$
\alpha=\int_{0}^{t} A \mathrm{e}^{-\frac{E}{R T}} \alpha^{m}(1-\alpha)^{n}
$$

Therefore, the results of this study is of great importance from the practical point.
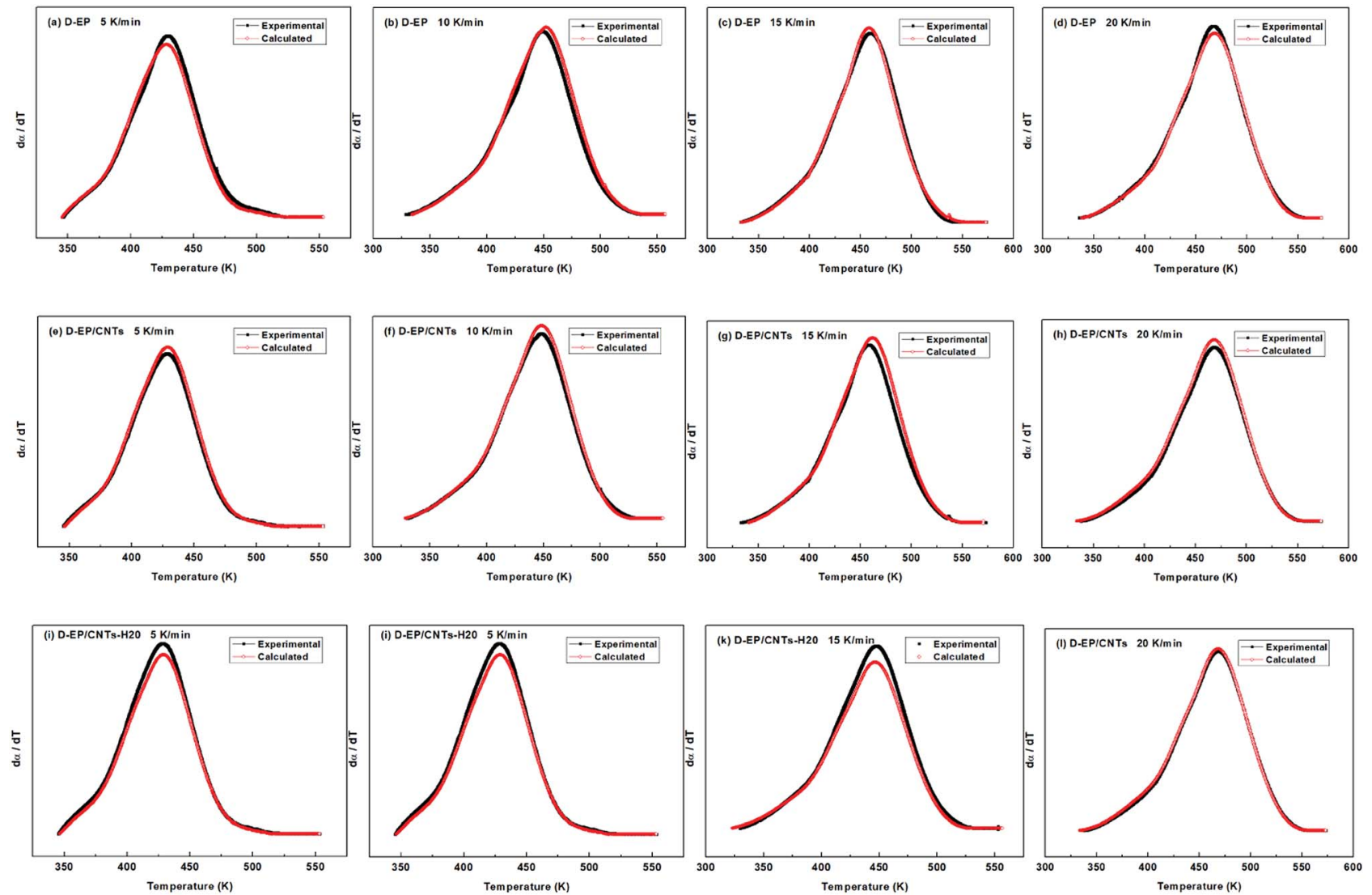

Fig. 9 Comparison of experimental values and calculated values at different heating rates for the D-EP, D-EP/CNTs and D-EP/CNTs-H2O systems. 


\section{Conclusions}

The cure kinetics of D-EP before and after the addition of CNTs or CNTs-H20 were investigated by non-isothermal DSC. It was found that the presence of CNTs shifted the cure temperature to lower temperature and accelerates the curing of D-EP. The addition of CNTs-H20 exhibited a stronger effect in accelerating the cure of D-EP.

Activation energy was calculated based on Kissinger approach and Ozawa approach. The lowered activation energy was observed after the addition of CNTs or CNTs-H20 at low degrees of cure, which indicated that the CNTs has a large effect on the curing reaction. The presence of CNTs facilitated the curing reaction, especially the initial epoxyamine reaction. Moreover, functionalized CNTs (CNTs-H20) exhibited better performance.

Autocatalytic model was used to describe the cure kinetic phenomena of studied systems. When the CNTs or CNTs-H20 was added, Sesták-Berggren model still can describe the cure kinetic of D-EP composites because the results, calculated by the model, agree with the experimental data well. Moreover, the kinetics parameters as well as the equation describing the cure process were proposed based on the Sesták-Berggren model. In general, the functionalized CNTs (CNTs-H20) exhibited obviously better performance in accelerating the cure of D-EP.

\section{Conflicts of interest}

There are no conflicts to declare.

\section{Acknowledgements}

We gratefully acknowledge the financial support from the National Natural Science Foundation of China (51702282), and the Foundation of Distinguished Young Scholars of Chongqing (CSTC2014JCYJJQ0019).

\section{References}

1 A. Boukerrou, J. Duchet, S. Fellahi and H. Sautereau, Processing of mica/epoxy nanocomposites by ultrasound mixing, J. Appl. Polym. Sci., 2007, 105(3), 1420-1425.

2 X. Luo, X. Yu, Y. Ma, K. Naito and Q. Zhang, Preparation and cure kinetics of epoxy with nanodiamond modified with liquid crystalline epoxy, Thermochim. Acta, 2018, 663, 1-8.

3 T. Na, H. Jiang, L. Zhao and C. Zhao, Preparation and characterization of novel naphthyl epoxy resin containing 4-fluorobenzoyl side chains for low-k dielectrics application, RSC Adv., 2017, 7(85), 53970-53976.

4 M. Qi, Y.-J. Xu, W.-H. Rao, X. Luo, L. Chen and Y.-Z. Wang, Epoxidized soybean oil cured with tannic acid for fully biobased epoxy resin, RSC Adv., 2018, 8(47), 26948-26958.

5 W. Li, D. Xiang, L. Wang, E. Harkin-Jones, C. Zhao, B. Wang and Y. Li, Simultaneous enhancement of electrical conductivity and interlaminar fracture toughness of carbon fiber/epoxy composites using plasma-treated conductive thermoplastic film interleaves, $R S C A d v .$, 2018, 8(47), 26910-26921.

6 A. Reghunadhan, J. Datta, N. Kalarikkal, J. T. Haponiuk and S. Thomas, Toughness augmentation by fibrillation and yielding in nanostructured blends with recycled polyurethane as a modifier, Appl. Surf. Sci., 2018, 442, 403411.

7 S. Fatemeh, A. Laura, P. Paola, C. Cosimo, B. Thatiane, C. M. O. Hilário, P. Concetto, S. Filippo and A. Veronica, A novel hybrid linear-hyperbranched poly(butylene adipate) copolymer as an epoxy resin modifier with toughening effect, Polym. Int., 2016, 65(3), 308-319.

8 Z. Hao, L. Li, X. Liao, X. Sheng and Y. Zhang, Preparation and toughening performance investigation of epoxy resins containing carbon nanotubes modified with hyperbranched polyester, Polym. Bull., 2018, 75(3), 10131026.

9 S. Jung-Hoon, P. B. Gangadhara and C. Alan, Effect of the addition of multi-walled carbon nanotubes on the thermomechanical properties of epoxy resin, Polym. Compos., 2017, 38(9), 1873-1880.

10 T. Tsafack, J. M. Alred, K. E. Wise, B. Jensen, E. Siochi and B. I. Yakobson, Exploring the interface between singlewalled carbon nanotubes and epoxy resin, Carbon, 2016, 105, 600-606.

11 W. Jianqiao, G. Jian, Z. Qingjie, G. Liang, L. Hao, D. Huoying, J. Wenge, S. Gang and Y. Xiaoping, Effect of different amino functionalized carbon nanotubes on curing behavior and mechanical properties of carbon fiber/epoxy composites, Polym. Compos., 2016, 24142.

12 A. M. Visco, A. Pistone, V. Brancato, D. Iannazzo and M. Fazio, Mechanical and physical properties of epoxy resin based nanocomposites reinforced with polyamine functionalized carbon nanotubes, Polym. Compos., 2016, 37(4), 1007-1015.

13 Y. Chen, S. Zhou, H. Zou and M. Liang, Investigation of cure kinetics of epoxy resin/organic montmorillonite system by differential scanning calorimetry, Polym. Sci., Ser. B, 2014, 56(5), 623-631.

14 J. Wu, J. Guo, Q. Zhang, L. Gao, H. Li, H. Deng, W. Jiang, G. Sui and X. Yang, Effect of different amino functionalized carbon nanotubes on curing behavior and mechanical properties of carbon fiber/epoxy composites, Polym. Compos., 2018, 39(S2), E733-E744.

15 A. Dettlaff, M. Sawczak, E. Klugmann-Radziemska, D. Czylkowski, R. Miotk and M. Wilamowska-Zawłocka, High-performance method of carbon nanotubes modification by microwave plasma for thin composite films preparation, RSC Adv., 2017, 7(51), 31940-31949.

16 M. Rajabi, K. Mahanpoor and O. Moradi, Removal of dye molecules from aqueous solution by carbon nanotubes and carbon nanotube functional groups: critical review, RSC Adv., 2017, 7(74), 47083-47090.

17 W. Han, X. Liao, Q. Yang, G. Li, B. He, W. Zhu and Z. Hao, Crystallization and morphological transition of poly(llactide)-poly(e-caprolactone) diblock copolymers with 
different block length ratios, $R S C A d v ., 2017,7(36), 22515-$ 22523.

18 J. Kang, J. He, Z. Chen, F. Yang, J. Chen, Y. Cao and M. Xiang, Effects of $\beta$-nucleating agent and crystallization conditions on the crystallization behavior and polymorphic composition of isotactic polypropylene/multi-walled carbon nanotubes composites, Polym. Adv. Technol., 2015, 26(1), 32-40.

19 J. Kang, J. H. He, Z. Chen, H. Yu, J. Chen, F. Yang, Y. Cao and M. Xiang, Investigation on the crystallization behavior and polymorphic composition of isotactic polypropylene/multiwalled carbon nanotubes composites nucleated with $\beta$ nucleating agent - the role of melt structures, J. Therm. Anal. Calorim., 2015, 119(3), 1769-1780.

20 H. Jiang, C. Li, T. Sun and J. Ma, A green and high energy density asymmetric supercapacitor based on ultrathin $\mathrm{MnO} 2$ nanostructures and functional mesoporous carbon nanotube electrodes, Nanoscale, 2012, 4(3), 807-812.

21 Z. Hao, Y. Tan, X. Zhang and F. Zhang, Epithermal ageing mechanism of gussasphalt, J. Wuhan Univ. Technol., Mater. Sci. Ed., 2009, 24(3), 466-470.

22 M. R. Saeb, E. Bakhshandeh, H. A. Khonakdar, E. Mader, C. Scheffler and G. Heinrich, Cure kinetics of epoxy nanocomposites affected by MWCNTs functionalization: a review, TheScientificWorldJournal, 2013, 2013, 703708.

23 W. Ye, W. Li, Q. Sun, J. Yu and Q. Gao, Microwave absorption properties of lightweight and flexible carbon fiber/magnetic particle composites, RSC Adv., 2018, 8(44), 24780-24786.

24 C. Yu, W. Gong, J. Zhang, W. Lv, W. Tian, X. Fan and Y. Yao, Hot pressing-induced alignment of hexagonal boron nitride in SEBS elastomer for superior thermally conductive composites, RSC Adv., 2018, 8(45), 25835-25845.

$25 \mathrm{M}$. Al Samman and W. Radke, Two-dimensional chromatographic separation of branched polyesters according to degree of branching and molar mass, Polymer, 2016, 99, 734-740.

26 R. d'Arcy, J. Burke and N. Tirelli, Branched polyesters: Preparative strategies and applications, Adv. Drug Delivery Rev., 2016, 107, 60-81.
27 J. Zhang and C. P. Hu, Synthesis, characterization and mechanical properties of polyester-based aliphatic polyurethane elastomers containing hyperbranched polyester segments, Eur. Polym. J., 2008, 44(11), 3708-3714.

28 J. Wang, R. Xu, F. Yang, J. Kang, Y. Cao and M. Xiang, Probing influences of support layer on the morphology of polyamide selective layer of thin film composite membrane, J. Membr. Sci., 2018, 556, 374-383.

29 R. Xu, G. Xu, J. Wang, J. Chen, F. Yang, J. Kang and M. Xiang, Influence of l-lysine on the permeation and antifouling performance of polyamide thin film composite reverse osmosis membranes, RSC Adv., 2018, 8(44), 25236-25247.

30 B. Xiong, R. Chen, F. Zeng, J. Kang and Y. Men, Thermal shrinkage and microscopic shutdown mechanism of polypropylene separator for lithium-ion battery: In-situ ultra-small angle X-ray scattering study, J. Membr. Sci., 2018, 545, 213-220.

31 B. Xiong, J. Kang, R. Chen and Y. Men, Initiation of cavitation upon drawing of pre-oriented polypropylene film: in situ SAXS and WAXD studies, Polymer, 2017, 128, 57-64.

32 W. J. Choi, R. L. Powell and D. S. Kim, Curing behavior and properties of epoxy nanocomposites with amine functionalized multiwall carbon nanotubes, Polym. Compos., 2009, 30(4), 415-421.

33 K. Yang, M. Gu and Y. Jin, Cure behavior and thermal stability analysis of multiwalled carbon nanotube/epoxy resin nanocomposites, J. Appl. Polym. Sci., 2008, 110(5), 2980-2988.

34 S. Kumar, S. K. Samal, S. Mohanty and S. K. Nayak, Study of curing kinetics of anhydride cured petroleum-based (DGEBA) epoxy resin and renewable resource based epoxidized soybean oil (ESO) systems catalyzed by 2methylimidazole, Thermochim. Acta, 2017, 654, 112-120.

35 A. Moroni, J. Mijovic, E. M. Pearce and C. C. Foun, Cure kinetics of epoxy resins and aromatic diamines, J. Appl. Polym. Sci., 1986, 32(2), 3761-3773. 